\title{
Developing Model for library Ontology Using Protégé Tool: Process, Reasoning and Visualisation
}

\author{
USMAN HAFIZ UBA, BASHIR SALISU ABUBAKAR And \\ MULIKATU YAKUBU IBRAHIM \\ Kano University of Science and Technology, Wudil
}

Library information system on web in based on HTML which can display information simply, mostly restricted to manual keyword searches which may results in irrelevant information retrieval. Semantic web overcome this issue, but cannot do without ontology. In this paper education sector is considered and library ontology is presented to pivot the role of library information system that will ensure the use and re-used knowledge and shared common understanding of library information system, which can be communicated between people and across application systems. The methodology is based on the tools used in developing the ontology, several tools are used to create ontology. This paper will discuss some terms of library through library ontology. And protégé tool with its extensible plug-in will be use to create the library ontology. Focusing on the facilities and services of a library, various aspects like super class and sub class hierarchy, creating a sub class, instances for class illustration, query retrieval process, visualization and graph views have been demonstrated.

Key-Words: - Semantic web, Ontology, Protégé tool.

\section{Introduction}

Most library information are now on web for storage and retrieval purpose, regarding to this specification of information domain is needed when retrieval of data, which could be achieved by web semantic. Semantic web [6] helps in creating data stores and writing rules for handling data on web. Semantic web therefore has to do with Ontology.

Ontology is a specification of conceptualization [5]. The tools used in creating Ontology include; swoop, Onto Editor, Oil, Onto Studio and Protégé. Indeed, in this paper a protégé tool was used in building our library ontology, because of it flexibility in terms of knowledge modelling.Much Ontology has been developed for semantic web in different aspects of knowledge. This study focus on how to develop library Ontology, which will consider library as the domain and the relationship among its attribute.

This paper is divided into six sections. The first section discuss on semantic web and Ontology, second section is the protégé tool, the third section contains literature review of the work, the proposed work details is in the fourth section, in the fifth section is the result and analysis of the proposed work, then lastly the sixth section contains the conclusion and the future work.

\section{PROBLEMS STATEMENT}

Library is one of the building structures of education that keeps the historic and future knowledge. Library as a domain gives basic idea which shows how classes are related to each other in ontology. Many research in knowledge management come with ontology that perform some library issues. 
Many researcher or developers creates ontology in different perspectives of library some shows how digital library services could be built from a repository, collections and metadata catalog [11]. As such one would not be able to add, edit or combine ontology concepts and include some other important concept related to library like equipment belongs to facility and reservation to services in library, to make library ontology complete.

Various types of ontology editors such as, Onto Editor, protégé, Onto studio, that allow researchers to build or edit their ontology, but protégé tool in this paper is considered and used to create our library ontology, more information about protégé is discussed in section two.

At present, research advances the use of ontology conducted by many researchers. For example, a collaborative knowledge management using ontology [14]. Library resources semantization base on resource ontology (OR) [15]. Ontology-based framework for sharing and integrating educational resource [16]. Subhasis [13] propose solution to conflict between role hierarchy and concept hierarchy in digital library ontology. And also Marcos [11] defines a formal, axiomatic ontology for digital library.

The research takes a new dimension considering the importance of semantic webs, ontology and the protégé tool to provide an agreed understanding of library as the main domain and other components of library, as its attributes and entities to establish relationship among them to come up with an agreed knowledge of information system on web with the help of the proposed library ontology, which will be illustrated in detail in section three.

\subsection{Semantic Webs}

Web is seen as a place where data are retrieved, saved and processed for information purposed from different source to different destination (consumers). And these data is been used almost every minutes and it keeps increasing which leads to a problem of data assembling and unfavourable to get complete information or to re use the data. The idea of semantic web [2] as an extension of the web by it initiator "Tim Burrners Lee", gives the solution to that problem, as it now provides information that is well define, has meaning and re-usable[3]. As Naveen Malviya et al expresses "it is the idea of having data on the web defined and linked in a way that it can be used for more effective discovery, common understanding of a particular knowledge across various application".

Although semantic web are not replacing the World Wide Web rather is an extension of the normal web used [7]. .

The target of semantic web is to provide a comprehensive linkage of any information on the web.

\subsection{Ontology}

Ontology is the backbone of the semantic web because ontology deals with the domain understanding, conceptualization [1] and agreed knowledge of the domain. Ontology gives a specific and common understanding based on the different concept of a domain. According to Tom "it is a set of representational primitives with which to model a domain of knowledge or discursion, the primitives are classes, attributes, and relationship" [5].ontology helps a researcher to come up with a specific and generic knowledge about a domain of study, it enable interoperability among web applications [4], because ontologies are independent from the application that uses them [3].

The language that is best used in developing any ontology is the OWL language, it is the one most developer of ontology use among other languages such as; XOL, SHOE, OML, RDF(s), DAML+OIL. Out of $100 \%$ of ontology creators, $75 \%$ of them uses OWL language, [3].Owl 
language is a new version of the existing language (DAML+OIL)[6], due to the addition of extra features to it to meet the need of a web ontology language

In this paper we focus on how protégé can be used to create ontology in owl language, and this approach will be shown in creating library ontology base on facility and service relationship using owl visualization and DL query plug-in of protégé. HermiT reasoner is used here for checking whether or not the ontology is properly identified or consistent.

\subsection{Protégé Tool}

Protégé tool is software that is use to create ontology and data entry about a domain ontology, it is produce by Stanford University and allow the description of classes, class hierarchy, entities, object property, data property, variables, variable hierarchy, relationship between classes and the relationship between properties. Protégé tool is available free online for download it is of different version and plug-in(s). Protégé comes with a reasoned that makes sure the domain ontology is proper, also a visualization plug-in such as Owl-Viz, Onto-graph, that allow the user to view the ontology diagram.

Protégé has two main ways to model an ontology, protégé-Frames and protégé-OWL, and protégé can save and export any ontology build in RDF, RDFS or OWL format [8].

\section{PROPOSED MODEL}

The ontology is developed in OWL language, using protégé tool of version 4.3. The proposed work is illustrated under six to seven simple steps as follows.

\section{STEP -I}

Classes and Classes hierarchy

This step gives the library ontology classes or concepts that would be considered for the work and illustrated in figure 3.

\section{STEP-II}

Object properties of ontology

Our object properties are base on the relationship that we want to add between classes. Figure 3 illustrate the relationship among the individuals.

\section{STEP-III}

Data properties of ontology

In this step, data properties of library ontology that shows the relationship between instance, individual and data value is provided, as it is shown in figure 5.

\section{STEP-IV}

Properties and relationship

Here we need to define the link that is in between the classes (like object properties) we use property which indicates the relationship between instances (individuals) as shown in figure 6. For example, (property) facility contains space, that is, space can be found among the library facilities.

The next is the data property which contains link between individuals, instances or data values such as (capacity, value). Which space has the capacity to accommodate five users?

Below is the object properties Domain and range example;

$<$ owl: Object property rdf: ID = "cotain"/>

$<$ rdfs: domain rdf: resource $=$ "\#facility" $/>$

$<$ rdfs: range rdf: resource $=$ "\#space" $/>$

$</$ owl: Object property>

\section{STEP-V}

The individuals (instances) of ontology 
When giving individual (instance), select the right class to which it belong, it can belong to more than one class. The rdf: type was used to select the class to which the individual belong, for example;

$</$ owl: thing rdf: id = "carrel"

$<$ rdf: type rdf: resource $=$ "\#space" $>$

$<$ rdf: type rdf: resource $=$ "\#borrow" $>>$

$</$ owl: thing $>$

\section{STEP-VI}

Reasoning the ontology

Reasoning the ontology makes it consistence and reliable, it is very important to use the reasoner because it check consistency and redundancy of knowledge. Reasoner makes the ontology to work well.

\section{RESULTS AND ANALYSIS}

In this section of the paper we discuss the result and analysis of our work (library ontology) based on the 'visualization view' of the class concept and the 'Query processes' for queering information about any individual.

\subsection{Visualization View}

This is where we show a display of our ontology, we include important classes and subclasses to our ontology. The visualization is capable with the help of the OWLVIZ[12], which is a plug-in found in the protégé tool and the display it made is in two view; the asserted view which gives the view of classes graph define in the ontology and the inferred view displays after reasoned the protégé tool base on our relationship in the ontology.

Below are some visualization result of our library ontology.

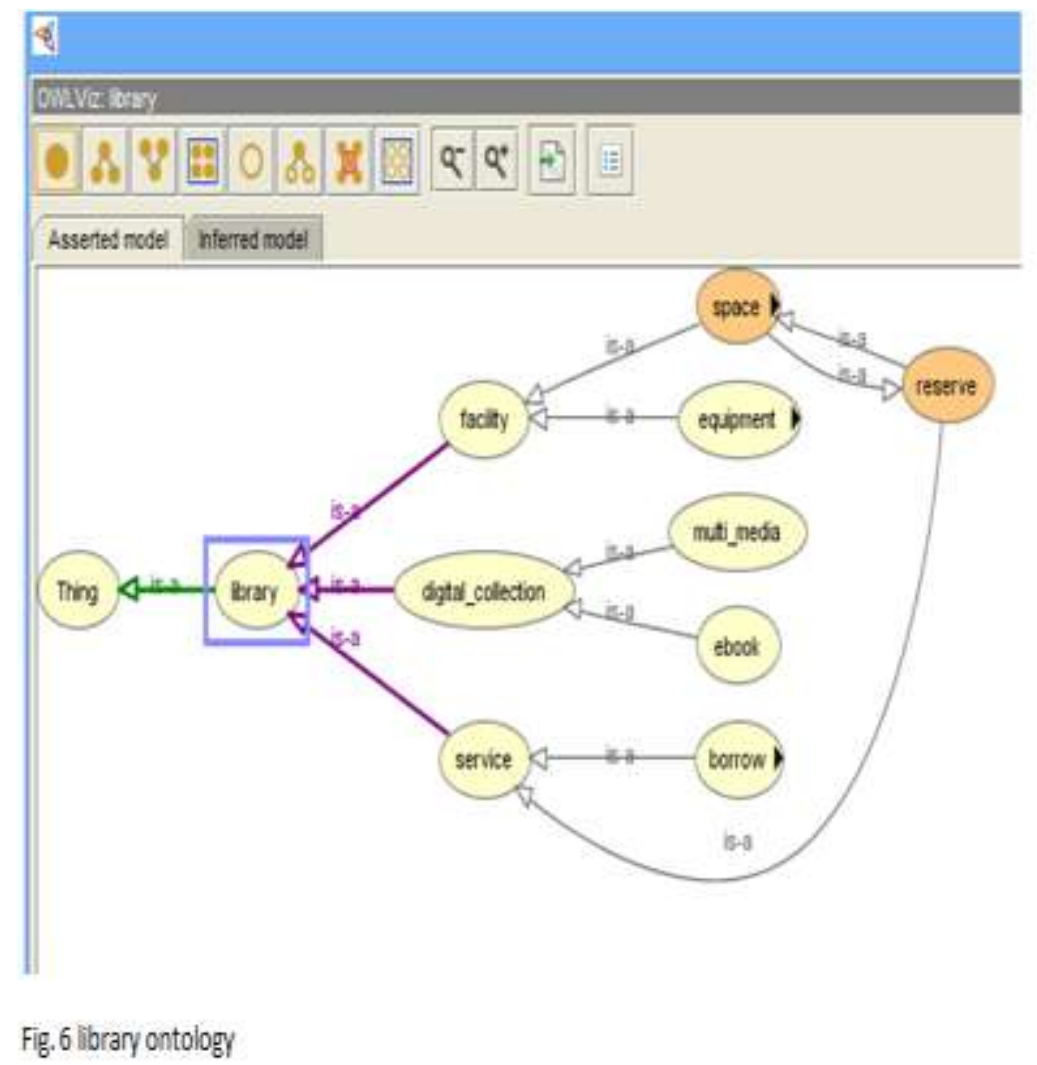


The above (figure 6) indicates two types of classes, the ones in yellow colour and the ones in orange. The orange colour classes are defined class that indicates sufficient condition, reserve and space are the defined class. The yellow colour are the primitive class which fulfil a necessary condition, the primitive classes includes; service, facility, equipment and so on.

Arrow within classes such as; space, equipment and borrow shows that there are subclasses and equivalent which are hidden in such classes.

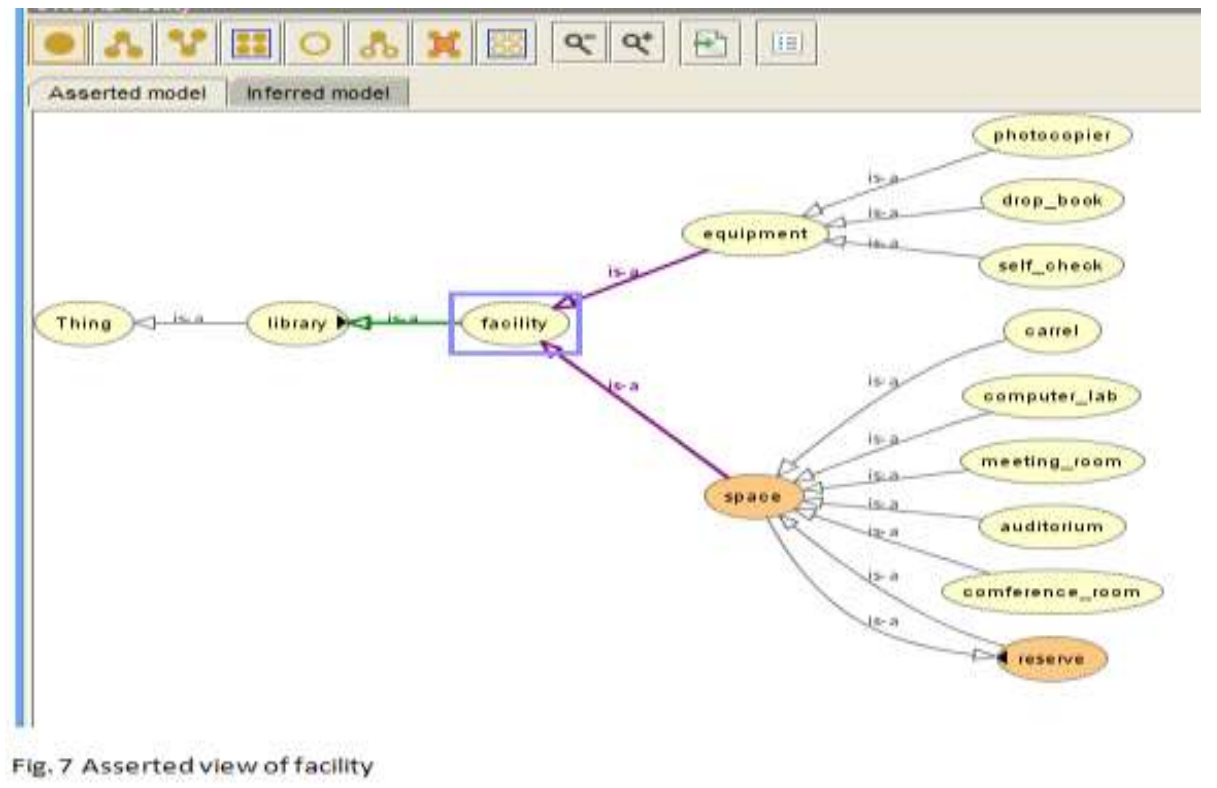

In this view (figure 7) facility base relationship is displayed. Thing is the supper class followed by library as its subclass, it contain some facilities which can be in form of space and equipment, then space and equipment are subclasses of facility concept, such as auditorium, carrel, meeting room are space part of facility while drop-books, self-check, photocopier are equipment part of the facility in the ontology.

Reserve class shows backside arrow which indicates that it is a subclass of some other class, and it has double is-a relationship due to the equivalent relation it has with space class. Every space in library as facility can be reserve to users.

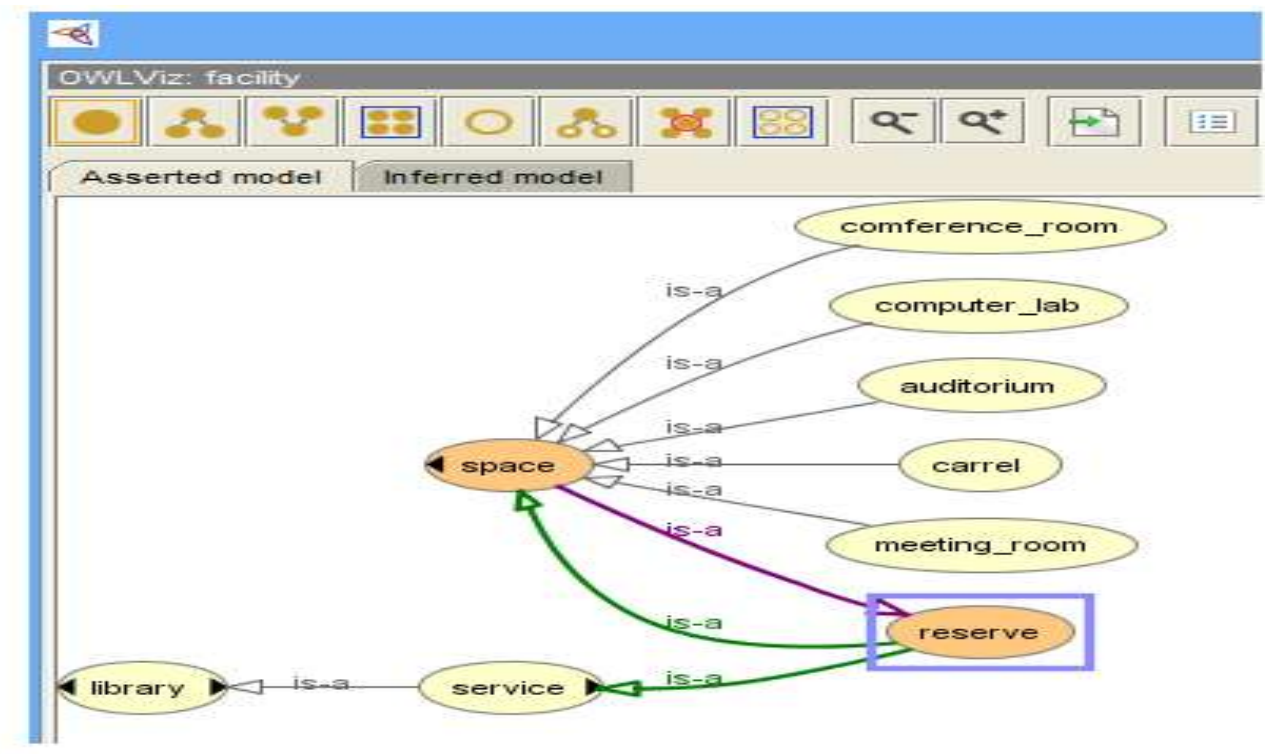

Fig. 8 An inferred view of facility base 
In the inferred view (as in figure 8), as defined earlier, space class is related to reserve class, this also relate all subclass of space to the reserve class. And both subclass of space are related to service concepts, because reserve class is related to service concept.

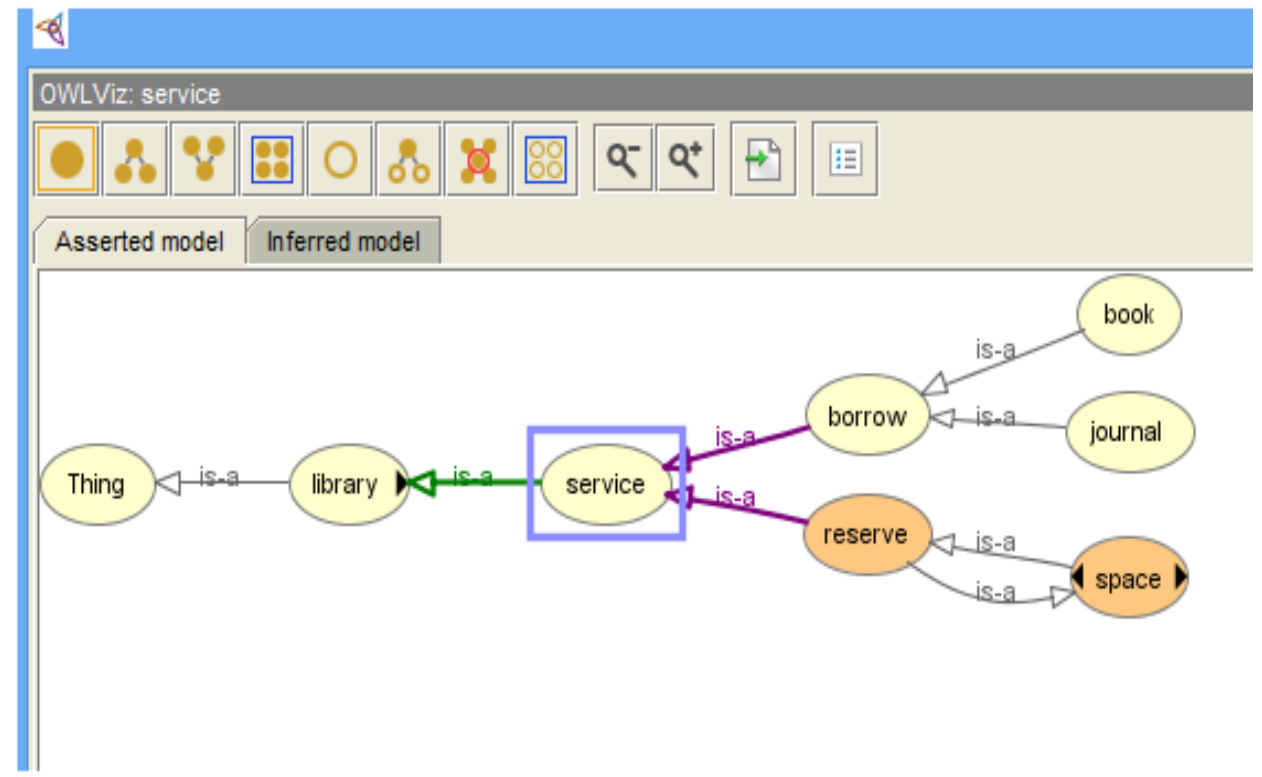

Fig. 9 Asserted view of service base

Library has several services it delivered, among is reserve and borrow (loan). In case of borrow as in (figure 9) above provides the subclasses book and journal. In the case of reserve it is related to space class which has subclasses such as; auditorium, meeting room, conference room, which the library can reserve for the users, based on which we can search for available space.

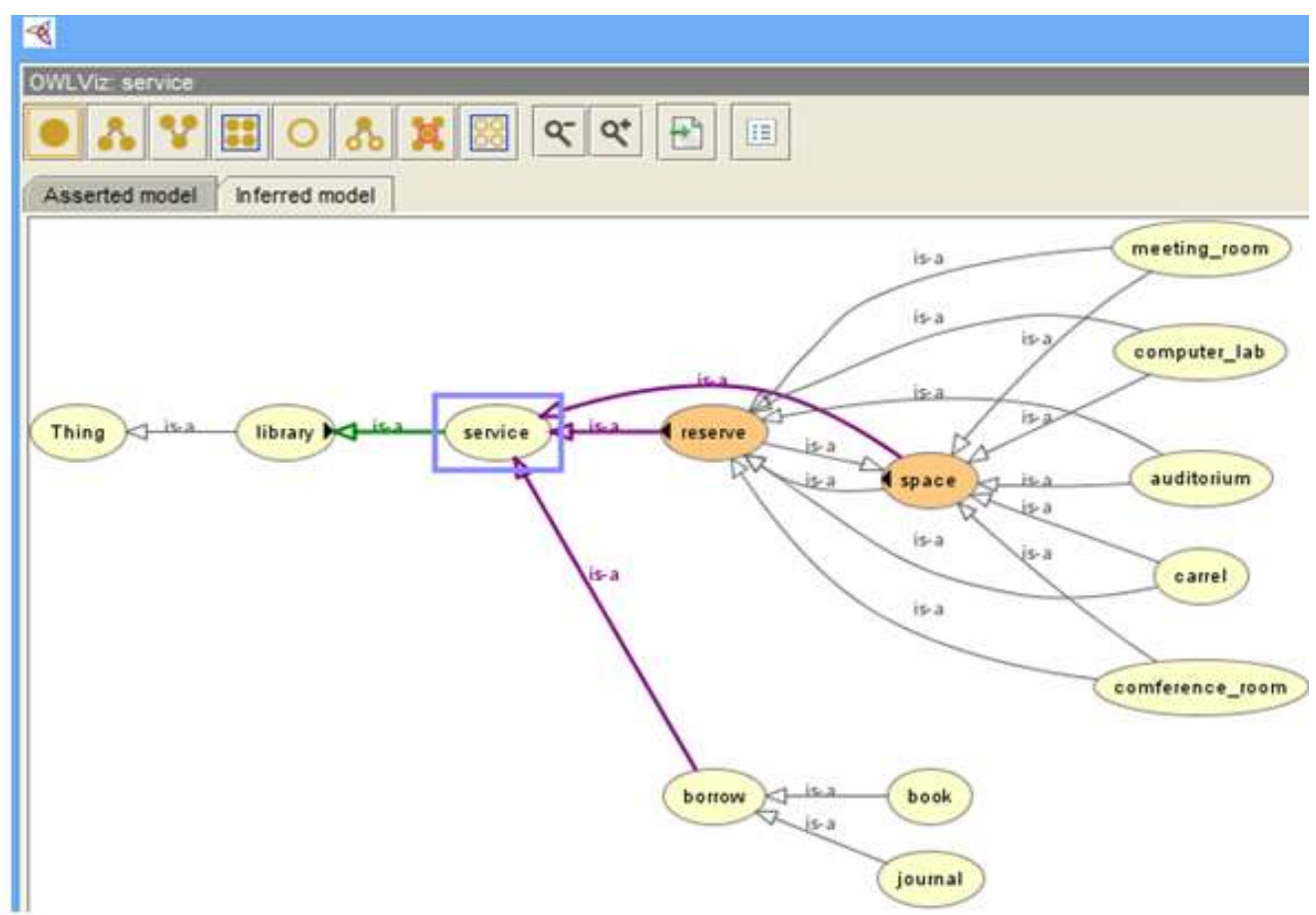

Fig. 10 An inferred view of service base 
In the inferred view (as in figure 10) above, all the subclasses of space class are related to the reserve class which is closely related to service concept. That is library service can be a provision of space like conference room etc.

\subsection{Query Process}

In order to query the database, DL Query plug-in of the protégé 4.3 version is used for searching of any information about our ontology. And this is done by entering the data property, object property or class name of the class information searching for.

But we make sure that the reasoner is started and being reasoning, else nothing will be executed.

The reasoner is started by clicking on the reasoner button on the top menu bar of the protégé tool, in the option box opened select "start reasoner", then if everything is normal the search will be processed and result will or related information about our search be displayed as shown in (figure 11).

For example, $\mathrm{f}$ we want to search for a meeting room that will contain ten people, simply type the data property of such class "capacity" follow by value. And result of the detail information will be displayed.

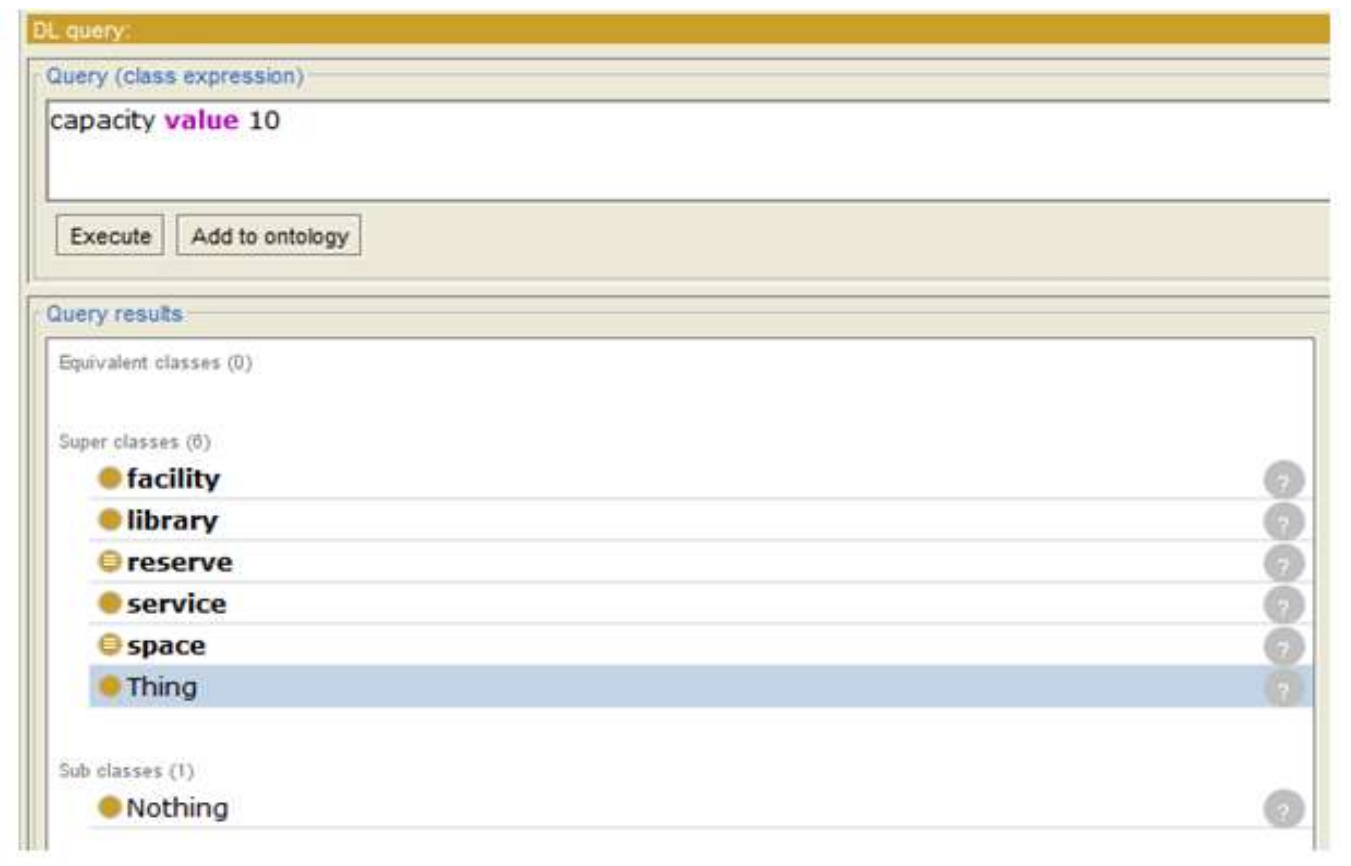

Fig. 11 a space querv result

\section{CONCLUSION}

This paper explained the design and development of our ontology which will be important in sharing common understanding of knowledge or information both for human or machine benefit which cloud enable interoperability, the ontology confirm the semantic web to made machine to agree and understand a specific knowledge and help people to get a good and sufficient result.

We focus to use the latest version of protégé tool which is widely and commonly used in developing ontology, and also we consider and use its important plug-in such as; class tab, object property tab, DL Query, OWLViz and so on. The research future work will be based on how to use the SPARQL Query on our library ontology. 
Acknowledgement:The authorwould like to thank the kano state government for sponsoring the study under kano state 502 foreign training.

\section{References:}

[1] Ontology definition by Tom Gruber:www.tomgruber.org

[2] Jorge Cardoso and Amit P. Sheth (Eds), semantic web, processes ande application, 2009.

[3] R. B. Mishra. Sandeep Kumar, Semantic web reasoner and languages, Artificial intelligent review, 2011, 35:339-368

[4] Mohammad Mustapha Taye, Understanding semantic web and ontology, journal of computing, Vol.2, ISSN2151-9617, (2010), pp.155

[5]www.tomgruber.org/writing/ontology_defination

[6] Semantic web definition: www.w3.org/standards/semanticweb

[7] Tracy V. Wilson, How semantic work:www.computer.howstuffworks.com/semantic_web

[8] Protégé tool for ontology: www.w3.org/protege

[9] Daniel L. Rubin. Natalya F. Noy, Mark A. Musen, "protégé: A tool for managing and using Terminology in radiology Application", Journal of digital library, Vol.20 (suppl 1); 2007, PMC2039856

[10] Emhimed Alatrish, comparison of ontology editors, Management information system, Vol. 8, No.2, 2013, pp 018-024.

[11] Marcos A. Goncalves. Edward A. Fox. Layne T. Watson, toward a digital library: a formal digital library ontology, International journal of digital library, Vol. 8, 2008, ISSN. 0033-1, pp. 91-92.

[12] Protégé.standard.edu

[13] Subhasis Dasgupta. Aditya Bagchi, resolving conflicts between role hierarchy and concept hierarchy in a digital library ontology,IEEE, 2012, pp 443-446.

[14] Weronika T. Adrian. Antoni Ligeza. Grzegorz J. Nalepa. Krzysztof Kaczor, Distributed and collaborative knowledge management using an ontology-based system, International Fedration for Information Processing, 2014, pp112-130.

[15] Yu Fan. Qiu junping. Wen Liu, Library resources semantization based on resource ontology, the electronic library, vol.32, ISS 3, 2014, pp.341-362.

[16] Jing Xiong. Yungtong Liu. Wei Liu, Ontology-based integration and sharing of big data educational resource, IEEE Computer society, 2014, pp. 245-248. 